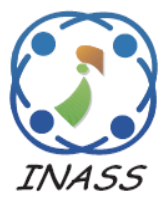

\title{
Modified Adaptive Sliding Mode Control for Trajectory Tracking of Mini-drone Quadcopter Unmanned Aerial Vehicle
}

\author{
Jutarut Chaoraingern ${ }^{1}$ \\ Vittaya Tipsuwanporn ${ }^{1}$ \\ Arjin Numsomran ${ }^{1 *}$ \\ ${ }^{I}$ Faculty of Engineering, King Mongkut's Institute of Technology Ladkrabang, Bangkok, Thailand \\ * Corresponding author's Email: arjin.nu@kmitl.ac.th
}

\begin{abstract}
Unmanned Aerial Vehicles are among the most widely attracting interest, especially in the applications of transportation, inspection, and surveillance. The great mechanisms for motion control are set to become a vital factor in performing the robust and accurate stabilized flight relied on perturbations and disturbances. This paper presents modified adaptive sliding mode control for trajectory tracking of mini-drone quadcopter unmanned aerial vehicles, which aims at demonstrating the effectiveness of nonlinear adaptive control strategy for achieving the desired performance of the mini-drone quadcopter system. Besides providing mathematical modeling and nonlinear dynamic characteristic details of mini-drone quadcopter actuated system, the modified adaptive sliding mode algorithm is developed using adaptation law based on Lyapunov stability approach then applied on the attitude loop and the altitude loop control system so that the nonlinear adaptive behavior of the controller enables the compensation of disturbances and parameter perturbations. The effectiveness validations of the proposed control technique compared with the traditional approach are performed through the Matlab simulation. The results have been illustrated that the modified adaptive sliding mode control can decrease the error performance indexes to the minimum ISE at $1.041 \mathrm{~m}^{2}$ and the zero percentage of overshoot while enables excellent stability and robustness even in the presence of parameter perturbations and disturbance.
\end{abstract}

Keywords: Adaptive sliding mode control, Parrot mini-drone, Trajectory tracking control, Quadcopter, Unmanned aerial vehicles.

\section{Introduction}

Over the past two decades, unmanned aerial vehicles (UAVs) are among the most widely attracting interest, especially in the applications of transportation, agriculture, inspection, and surveillance. Several studies have been conducted; for example, the economical drone was developed and applied to archaeological data collection projects for improving the data accuracy and survey efficiency in the field [1]. UAV included with the technology of close-range photogrammetry (CRP) was purposed for collecting the data of permanent deformation of cracking patterns in pavement infrastructure to illustrate the effective use and reliable assessments in the field conditions [2]. Lightweight unmanned aerial vehicles (UAVs) were applied as a tool for long-term ecological monitoring to explain local-scale variation in forest stand and species measures [3]. UAV with a multispectral camera was implemented in precision agriculture for monitoring the infected areas so that increasing crop productivity [4]. In [5], the application of UAV vaccine transportation was evaluated in terms of logistics cost savings and increase vaccine availability.
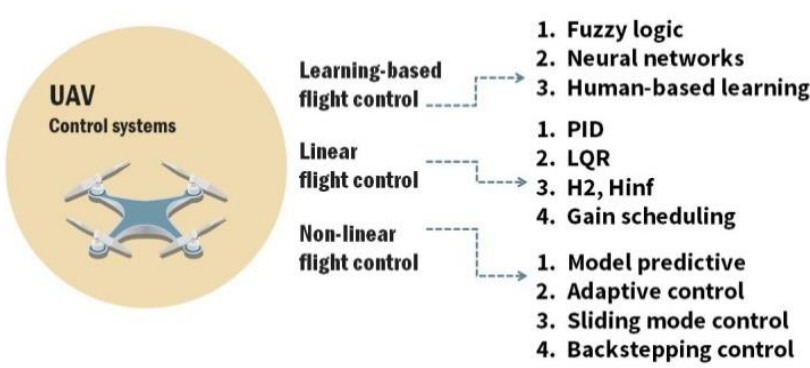

Figure. 1 Classification of control techniques developed for quadcopter UAV 
The great mechanisms for motion control are set to become a vital factor in performing the robust and accurate stabilized flight relied on perturbations and disturbances. The diverse control techniques implemented on quadcopter UAV can be classified into three categories: linear flight control, learningbased flight control, and nonlinear flight control [6]. The linear flight control is the one commonly used to control quadcopter motion due to the design and development of simple controller algorithms. Many studies, for instance [7, 8], were carried out to demonstrate the optimal solution of PID control and particle swarm optimization algorithm implemented on quadrotor altitude and attitude control system for increasing performance as well as robustness against external disturbances. In [9-11], linear quadratic regulator (LQR) was modified and applied to unmanned quadcopter for overcome the traditional PID control system that had the side-effect of linearization of a model while extended stability and robustness of trajectory tracking control. Learningbased flight control has been performed on UAV altitude and attitude control in order to deal with the complex nonlinear system, strong coupling, and various uncertainties. For example, in [12-14], the fuzzy logic nonlinear altitude control has illustrated the advantage of dealing with nonlinear, uncertainties, and under-actuated systems. In [15], the Sigma-Pi neural network was proposed in order to enhance the performance of position and attitude quadrotor control compared with the PID controller.

The nonlinear flight control has been suggested with the purpose of transcending the restrictions of linear controller that may fail to provide satisfactory control performance in a variety of operation ranges, as well as to require fewer complex computational tasks, likewise achieving more control performance and robustness. Model predictive control techniques for quadrotor prototypes have been implemented in $[16,17]$ to validate the efficiency of position tracking and attitude control. Sliding mode control for quadrotor attitude and position control has been proposed in [18-20] to improve the position tracking performance over traditional PID control.

Many studies of extended sliding mode control have been published to overcome the effects of chattering phenomenon, parameter uncertainties, and external disturbances. In [21, 22], sliding mode control with disturbance observer was proposed for rejecting the effect of the unknown disturbance in the quadrotor while achieving the performance of attitude control and disturbance suppression. In [23, 24] backstepping sliding mode control technique was presented to accomplish quadrotor altitude, attitude and trajectory tracking control performance.
The main contribution of this paper is to suggest a modified adaptive sliding mode control for trajectory tracking of mini-drone quadcopter unmanned aerial vehicles. In this study, we concentrate on the design of an effective nonlinear adaptive control law, which aims at demonstrating the effectiveness of nonlinear adaptive control strategy for enhancing the performance of minidrone quadcopter UAV motion control by compensating the effects of system uncertainties while reducing the percent overshoot. Besides providing mathematical modeling and nonlinear dynamic characteristic details of parrot mini-drone quadcopter actuated system, we introduce the modified adaptive sliding mode algorithm is developed using adaptation law based on Lyapunov stability approach then applied on the attitude loop and the altitude loop control system so that the adaptive gain of the controller enables the compensation of parameter perturbations and disturbance. The advantages of our approach have been demonstrated through the simulation results considering performance and robustness for altitude, and position tracking control compared with nonadaptive SMC, and conventional PID control.

The remainder of this paper is organized as follows: The mathematical modeling and nonlinear dynamic characteristic of the mini-drone quadcopter unmanned aerial vehicle are defined in section 2 . Section 3 describes the theoretical aspects of modified adaptive sliding mode control. The simulation results and discussion are demonstrated in section 4, while the conclusion is described in section 5 .

\section{Dynamic model of X-type mini-drone quadcopter}

Mini-drone quadcopter is a small type of UAV which has the whole weight of 0.1 kilogram and 6.5

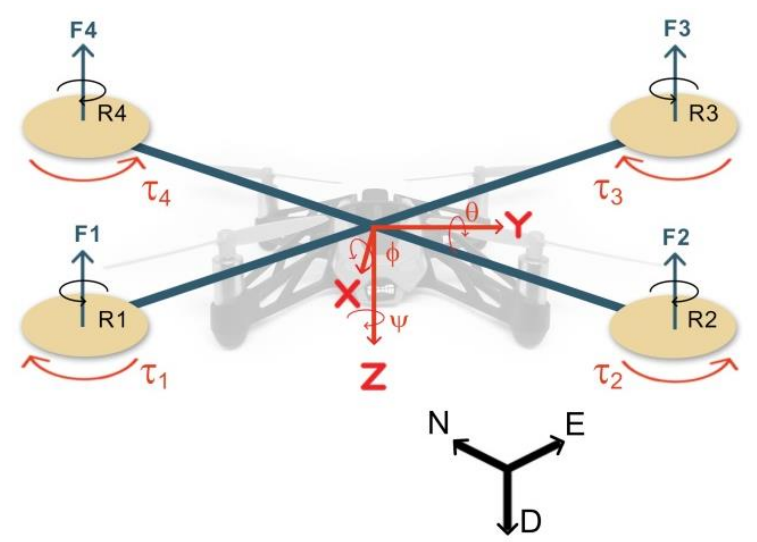

Figure. 2 Quadcopter reference frame and Euler angles 
centimeters of propeller size. It consists of four rotors installed at the end of the X-type structure, which the two front rotors and two rear rotors aligned with the $\mathrm{X}$-axis, as well as two left rotors and two right rotors aligned with the $\mathrm{Y}$-axis.

\subsection{Frames of reference}

In Fig. 2, two reference frames are used for demonstrating quadcopter flight motion. The inertial frame, North (N), East (E), and Down (D) axis, is the Earth frame on the specific ground level as well as the body frame, $\mathrm{X}, \mathrm{Y}$, and $\mathrm{Z}$-axis, is the frame originated at the center of the quadcopter body. In the X-type structure, $\mathrm{X}$-axis points toward the center point between rotor 1 and rotor 2 , and $\mathrm{Y}$-axis points towards the center point between rotor 2 and rotor 3 while the $\mathrm{Z}$-axis points downward to the ground.

\subsection{Translational equation of motion}

Two subsystems, which are translational motion and rotational motion, will be defined as the dynamic motion of the quadcopter based on the Newton-Euler formulation [25]. The total forces and moments acting on the quadcopter will be investigated and utilized to express the formulation of the state variables.

The translational motion represented the relation of total forces, and quadcopter acceleration is expressed as

$$
\begin{gathered}
m\left[\begin{array}{c}
\ddot{x} \\
\ddot{y} \\
\ddot{z}
\end{array}\right]=\left[\begin{array}{c}
0 \\
0 \\
m g
\end{array}\right]+R_{e}\left[\begin{array}{c}
0 \\
0 \\
-T
\end{array}\right] \\
R_{e}=\left[\begin{array}{ccc}
c \theta c \psi & c \psi s \theta s \phi-c \phi s \psi & s \phi s \psi+c \phi c \psi s \theta \\
c \theta s \psi & c \phi c \psi+s \theta s \phi s \psi & c \phi s \theta s \psi-c \psi s \phi \\
-s \theta & c \theta s \phi & c \theta c \phi
\end{array}\right] \\
T=\sum_{i=1}^{4}\left(b \omega_{i}^{2}\right)
\end{gathered}
$$

where $c$ refers to cos, and $s$ refers to $\sin . R_{e}$ is the rotational matrix that transforms the thrust force from body frame to the inertial frame. $m$ is a mass of quadcopter, $\ddot{x}, \ddot{y}, \ddot{z}$ are acceleration corresponding to the translational positions in the inertial frame, $g$ is constant of gravitation, $T$ is the total thrust force created by the four propellers. $b$ is the thrust constant, $\omega_{i}$ is angular velocity $(\mathrm{rad} / \mathrm{s})$.

The acceleration along the $\mathrm{N}, \mathrm{E}$, and $\mathrm{D}$ axis can be represented as

$$
\begin{aligned}
& m\left[\begin{array}{c}
\ddot{x} \\
\ddot{y} \\
\ddot{z}
\end{array}\right]=\left[\begin{array}{c}
0 \\
0 \\
m g
\end{array}\right]+ \\
& {\left[\begin{array}{ccc}
c \theta c \psi & c \psi s \theta s \phi-c \phi s \psi & s \phi s \psi+c \phi c \psi s \theta \\
c \theta s \psi & c \phi c \psi+s \theta s \phi s \psi & c \phi s \theta s \psi-c \psi s \phi \\
-s \theta & c \theta s \phi & c \theta c \phi
\end{array}\right]\left[\begin{array}{c}
0 \\
0 \\
-T
\end{array}\right]}
\end{aligned}
$$

From Eq. (4), the accelerations of quadcopter are given by

$$
\begin{aligned}
& \ddot{x}=-\frac{T}{m}[\cos (\phi) \sin (\theta) \cos (\psi)+\sin (\phi) \sin (\psi)] \\
& \ddot{y}=-\frac{T}{m}[\cos (\phi) \sin (\theta) \sin (\psi)-\sin (\theta) \cos (\psi)] \\
& \ddot{z}=-\frac{T}{m}[\cos (\phi) \cos (\theta)]+g
\end{aligned}
$$

\subsection{Rotational equation of motion}

The conservation of angular momentum in terms of moments of inertia can be expressed by using Euler's rotation equations describing the threedimensional rotation of a mini-drone quadcopter as

$$
\begin{aligned}
& I \cdot \dot{\omega}=-\omega \times(I \cdot \omega)-M_{g h}+M_{B} \\
& I=\left[\begin{array}{ccc}
I_{x x} & 0 & 0 \\
0 & I_{y y} & 0 \\
0 & 0 & I_{z z}
\end{array}\right] \\
& M_{g h}=\left[\begin{array}{c}
-J_{r} \Omega_{r} \dot{\theta} \\
J_{r} \Omega_{r} \dot{\phi} \\
0
\end{array}\right] \\
& M_{B}=\left[\begin{array}{c}
M_{x} \\
M_{y} \\
M_{z}
\end{array}\right]
\end{aligned}
$$

where 


$$
\begin{aligned}
& M_{x}=l b\left(\omega_{1}^{2}-\omega_{2}^{2}-\omega_{3}^{2}+\omega_{4}^{2}\right) \\
& M_{y}=l b\left(\omega_{1}^{2}+\omega_{2}^{2}-\omega_{3}^{2}-\omega_{4}^{2}\right) \\
& M_{z}=d\left(-\omega_{1}^{2}+\omega_{2}^{2}-\omega_{3}^{2}+\omega_{4}^{2}\right)
\end{aligned}
$$

$I$ is quadcopter body's inertia tensor $\left(\mathrm{kg} \cdot \mathrm{m}^{2}\right)$. $\omega_{i}$ is angular velocity $(\mathrm{rad} / \mathrm{s})$.

$M_{g h}$ is the gyroscopic moment from rotors inertia $(N \cdot m)$.

$M_{B}$ refers to the moment caused by drag forces and thrust performed in $\mathrm{X}, \mathrm{Y}, \mathrm{Z}$ axis respectively $(N \cdot m)$.

$J_{r}$ is rotor inertia $\left(\mathrm{kg} \cdot \mathrm{m}^{2}\right)$.

$\Omega$ refers to the total residual angular speed of motors and $\Omega_{r}=\omega_{1}-\omega_{2}+\omega_{3}-\omega_{4}(\mathrm{rad} / \mathrm{s})$

The rotational equation of motion in Eq. (8) can be simplified and expressed by the total torque acting on quadcopter body as

$$
\begin{aligned}
& {\left[\begin{array}{ccc}
I_{x x} & 0 & 0 \\
0 & I_{y y} & 0 \\
0 & 0 & I_{z z}
\end{array}\right] \cdot\left[\begin{array}{c}
\ddot{\phi} \\
\ddot{\theta} \\
\ddot{\psi}
\end{array}\right]=-\left[\begin{array}{c}
\dot{\phi} \\
\dot{\theta} \\
\dot{\psi}
\end{array}\right] \times\left[\begin{array}{ccc}
I_{x x} & 0 & 0 \\
0 & I_{y y} & 0 \\
0 & 0 & I_{z z}
\end{array}\right]\left[\begin{array}{c}
\dot{\phi} \\
\dot{\theta} \\
\dot{\psi}
\end{array}\right]} \\
& -\left[\begin{array}{c}
J_{r} \Omega_{r} \dot{\theta} \\
-J_{r} \Omega_{r} \dot{\phi} \\
0
\end{array}\right]+\left[\begin{array}{c}
l b\left(\omega_{1}^{2}-\omega_{2}^{2}-\omega_{3}^{2}+\omega_{4}^{2}\right) \\
l b\left(\omega_{1}^{2}+\omega_{2}^{2}-\omega_{3}^{2}-\omega_{4}^{2}\right) \\
d\left(-\omega_{1}^{2}+\omega_{2}^{2}-\omega_{3}^{2}+\omega_{4}^{2}\right)
\end{array}\right] \\
& I_{x x} \ddot{\phi}=-\dot{\theta} \dot{\psi}\left(I_{z z}-I_{y y}\right)-j_{r} \Omega_{r} \dot{\theta} \\
& \quad+l b\left(\omega_{1}^{2}-\omega_{2}^{2}-\omega_{3}^{2}+\omega_{4}^{2}\right) \\
& I_{y y} \ddot{\theta}=-\ddot{\theta} \dot{\psi}\left(I_{x x}-I_{z z}\right)+J_{r} \Omega_{r} \dot{\phi} \\
& +l b\left(\omega_{1}^{2}+\omega_{2}^{2}-\omega_{3}^{2}-\omega_{4}^{2}\right) \\
& I_{z z} \ddot{\psi}=-\dot{\phi} \dot{\theta}\left(I_{y y}-I_{x x}\right) \\
& +d\left(-\omega_{1}^{2}+\omega_{2}^{2}-\omega_{3}^{2}+\omega_{4}^{2}\right)
\end{aligned}
$$

where

$d$ refers to drag constant.

$b$ refers to thrust constant.

$l$ is the lever length of the quadcopter's arms $(\mathrm{cm})$.

$\omega_{i}$ is the angular velocity of the quadcopter motors

$(\mathrm{rad} / \mathrm{s})$.
From Eq. (16-18), the angular acceleration of quadcopter $\ddot{\phi}, \ddot{\theta}, \ddot{\psi}$ are defined as

$$
\begin{aligned}
& \ddot{\phi}=\left[\dot{\theta} \dot{\psi}\left(I_{y y}-I_{z z}\right)+j_{r} \Omega_{r} \dot{\theta}+u_{2}\right] / I_{x x} \\
& \ddot{\theta}=\left[\ddot{\theta} \dot{\psi}\left(I_{z z}-I_{x x}\right)-J_{r} \Omega_{r} \dot{\phi}+u_{3}\right] / I_{y y} \\
& \ddot{\psi}=\left[\dot{\phi} \dot{\theta}\left(I_{x x}-I_{y y}\right)+u_{4}\right] / I_{z z}
\end{aligned}
$$

where

$$
\begin{aligned}
& u_{2}=l b\left(\omega_{1}^{2}-\omega_{2}^{2}-\omega_{3}^{2}+\omega_{4}^{2}\right) \\
& u_{3}=l b\left(\omega_{1}^{2}+\omega_{2}^{2}-\omega_{3}^{2}-\omega_{4}^{2}\right) \\
& u_{4}=d\left(-\omega_{1}^{2}+\omega_{2}^{2}-\omega_{3}^{2}+\omega_{4}^{2}\right)
\end{aligned}
$$

\subsection{Mini-drone quadcopter dynamic state equation}

\subsubsection{State vector}

As the aforementioned dynamic model of X-type mini-drone quadcopter, the state vector defining the position of the quadcopter, the corresponding velocity in the inertial frame as well as its Euler angle is expressed as

$$
X=[x(t) y(t) z(t) \phi(t) \theta(t) \psi(t)]^{T}
$$

where

$$
\begin{aligned}
& \ddot{x}=-\frac{1}{m} u_{x} u_{1} \\
& \ddot{y}=-\frac{1}{m} u_{y} u_{1} \\
& \ddot{z}=g-\frac{1}{m}[\cos (\phi) \cos (\theta)] u_{1} \\
& \ddot{\phi}=\left[\dot{\theta} \dot{\psi}\left(I_{y y}-I_{z z}\right)+J_{r} \Omega_{r} \dot{\theta}+u_{2}\right] / I_{x x} \\
& \ddot{\theta}=\left[\ddot{\theta} \dot{\psi}\left(I_{z z}-I_{x x}\right)-J_{r} \Omega_{r} \dot{\phi}+u_{3}\right] / I_{y y} \\
& \ddot{\psi}=\left[\dot{\phi} \dot{\theta}\left(I_{x x}-I_{y y}\right)+u_{4}\right] / I_{z z}
\end{aligned}
$$


$\phi, \theta, \varphi$ refer to roll, pitch and yaw Euler angles. $x, y, z$ refer to the distances from the center of mass of mini-drone to the origin of earth fixed inertial frame. $\dot{\phi}, \dot{\theta}, \dot{\varphi}$ refer to Euler angular rate. $\dot{x}, \dot{y}, \dot{z}$ refer to the velocity of quadcopter in inertial frame.

\subsubsection{Input vector}

$$
\begin{aligned}
& U=\left[\begin{array}{llll}
u_{1} & u_{2} & u_{3} & u_{4}
\end{array}\right]^{T} \\
& u_{1}=b\left(\omega_{1}^{2}+\omega_{2}^{2}+\omega_{3}^{2}+\omega_{4}^{2}\right) \\
& u_{2}=l b\left(\omega_{1}^{2}-\omega_{2}^{2}-\omega_{3}^{2}+\omega_{4}^{2}\right) \\
& u_{3}=l b\left(\omega_{1}^{2}+\omega_{2}^{2}-\omega_{3}^{2}-\omega_{4}^{2}\right) \\
& u_{4}=d\left(-\omega_{1}^{2}+\omega_{2}^{2}-\omega_{3}^{2}+\omega_{4}^{2}\right) \\
& u_{x}=\cos (\phi) \sin (\theta) \cos (\psi)+\sin (\phi) \sin (\psi) \\
& u_{y}=\cos (\phi) \sin (\theta) \sin (\psi)-\sin (\theta) \cos (\psi)
\end{aligned}
$$

The vector of control input $(U)$ consists of four inputs; $u_{1} \sim u_{4}$ which are the input percentage commands applied to 4 rotors $i=1,2,3,4$ respectively.

The dynamics state equation of mini-drone quadcopter can be described as

$$
\ddot{X}=F_{T}(\dot{X})+G_{T}(X) U
$$

where $F_{T}(\dot{X})$ and $G_{T}(X)$ refer to the total nonlinear dynamics of the mini-drone quadcopter system. $U$ is the input vector .

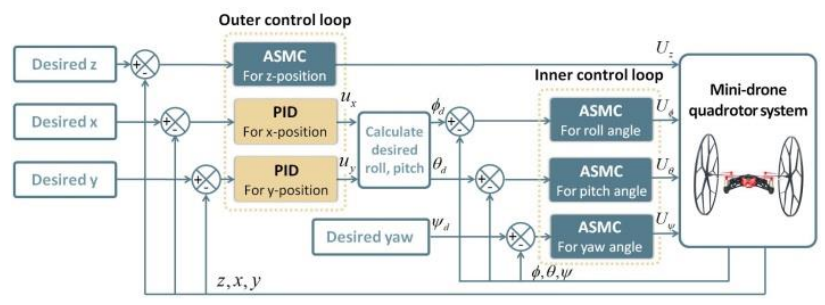

Figure. 3 Block diagram of modified adaptive sliding mode control for mini-drone quadcopter

$$
F(\dot{X})=\left[\begin{array}{l}
0 \\
0 \\
g \\
\dot{\theta} \dot{\psi} \frac{\left(I_{y y}-I_{z z}\right)}{I_{x x}}+\frac{J_{r}}{I_{x x}} \Omega_{r} \dot{\theta} \\
\dot{\theta} \dot{\psi} \frac{\left(I_{z z}-I_{x x}\right)}{I_{y y}}-\frac{J_{r}}{I_{y y}} \Omega_{r} \dot{\phi} \\
\dot{\phi} \dot{\theta} \frac{\left(I_{x x}-I_{y y}\right)}{I_{z z}}
\end{array}\right]
$$

$$
G(X)=\left[\begin{array}{llllll}
-1 / m & 0 & 0 & 0 & 0 & 0 \\
0 & -1 / m & 0 & 0 & 0 & 0 \\
0 & 0 & -\mathrm{c} \phi \mathrm{c} \theta / m & 0 & 0 & 0 \\
0 & 0 & 0 & 1 / I_{x x} & 0 & 0 \\
0 & 0 & 0 & 0 & 1 / I_{y y} & 0 \\
0 & 0 & 0 & 0 & 0 & 1 / I_{z z}
\end{array}\right]
$$

$$
U=\left[\begin{array}{l}
u_{1} u_{x} \\
u_{1} u_{y} \\
u_{1} \\
u_{2} \\
u_{3} \\
u_{4}
\end{array}\right]
$$

\section{Modified adaptive sliding mode control for mini-drone quadcopter}

The design of the modified adaptive sliding mode (MASMC) controller for the mini-drone quadcopter is described in this part. The block diagram in Fig. 3, depicts the proposed MASMC scheme. It can be seen that the MASMC has been applied to the inner loop of attitude control and the outer loop of altitude control. The aim is to design the nonlinear and robust controller, which enables efficient motion control performance, low overshoot, as well as has a capability of process uncertainty and disturbance rejection.

\subsection{Modified adaptive sliding mode control}

The MASMC consists of the equivalent control law $U_{E}$ which produces the control signal in a reaching phase and the adaptive switching control 
law $U_{A S}$ that produces the control signal in a sliding phase.

$$
U_{M A S M C}=U_{E}+U_{A S}
$$

A proportional-integral-derivative (PID) sliding surface is developed for determining the equivalent control law $U_{E}$.

$$
s(t)=K_{p} e(t)+K_{i} \int_{0}^{t} e(t) d t+K_{d} \dot{e}(t)
$$

where $K_{p}, K_{i}, K_{d} \in R^{+}$are controller gains. The derivative term of the sliding surface is shown as follows:

$$
\dot{s}(t)=K_{p} \dot{e}(t)+K_{i} e(t)+K_{d} \ddot{e}(t)
$$

The adaptive switching control law $U_{A S}$ is developed by the following adaptive sliding mode strategy for decreasing the chattering phenomenon during the occurrence of disturbance and system uncertainty.

$$
\begin{aligned}
& U_{A S}=-K_{a} \operatorname{sgn}(s)-\eta s \\
& \dot{K}_{a}=\left\{\begin{array}{cl}
K \operatorname{sgn}(|s|-\mu), & \text { if } K_{a}>K_{\mathrm{m}}, \\
K_{\min }, & \text { if } K_{a} \leq K_{\mathrm{m}}
\end{array}\right.
\end{aligned}
$$

where

$K_{a}$ is adaptation rate.

$\eta, K, \mu$ are positive constant.

$K_{\mathrm{m}}$ is the threshold of the adaptation.

\subsection{Attitude control law}

The attitude control law is formulated as follows to control the rotation of the mini-drone quadcopter [26].

\subsubsection{Roll control}

$$
\begin{aligned}
s_{\phi} & =K_{p \phi}\left(\phi_{d}-\phi\right)+K_{i \phi} \int_{0}^{t}\left(\phi_{d}-\phi\right) d t+K_{d \phi}\left(\dot{\phi}_{d}-\dot{\phi}\right) \\
\dot{s}_{\phi} & =K_{p \phi}\left(\dot{\phi}_{d}-\dot{\phi}\right)+K_{i \phi}\left(\phi_{d}-\phi\right)+K_{d \phi}\left(\ddot{\phi}_{d}-\ddot{\phi}\right) \\
& =-\varepsilon_{\phi} \operatorname{sgn} s_{\phi}-\eta_{\phi} s_{\phi}
\end{aligned}
$$

$$
\begin{aligned}
u_{2} & =\frac{I_{x x}}{K_{d \phi}} K_{p \phi}\left(\dot{\phi}_{d}-\dot{\phi}\right)+\frac{I_{x x}}{K_{d \phi}} K_{i \phi}\left(\phi_{d}-\phi\right)+I_{x x} \ddot{\phi}_{d} \\
& -\dot{\theta} \dot{\psi}\left(I_{y y}-I_{z z}\right)-J_{r} \Omega_{r} \dot{\theta}+\frac{I_{x x}}{K_{d \phi}} \varepsilon_{\phi} \operatorname{sgn} s_{\phi}+\frac{I_{x x}}{K_{d \phi}} \eta_{\phi} s_{\phi}
\end{aligned}
$$

\subsubsection{Pitch control}

$$
s_{\theta}=K_{p \theta}\left(\theta_{d}-\theta\right)+K_{i \theta} \int_{0}^{t}\left(\theta_{d}-\theta\right) d t+K_{d \theta}\left(\dot{\theta}_{d}-\dot{\theta}\right)
$$

$$
\begin{aligned}
\dot{s}_{\theta} & =K_{p \theta}\left(\dot{\theta}_{d}-\dot{\theta}\right)+K_{i \theta}\left(\theta_{d}-\theta\right)+K_{d \theta}\left(\ddot{\theta}_{d}-\ddot{\theta}\right) \\
& =-\varepsilon_{\theta} \operatorname{sgn} s_{\theta}-\eta_{\theta} s_{\theta}
\end{aligned}
$$

$$
\begin{aligned}
u_{3} & =\frac{K_{p \theta} I_{y y}}{K_{d \theta}}\left(\dot{\theta}_{d}-\dot{\theta}\right)+\frac{K_{i \theta} I_{y y}}{K_{d \theta}}\left(\theta_{d}-\theta\right)+I_{y y} \ddot{\theta}_{d} \\
& -\ddot{\theta} \dot{\psi}\left(I_{z z}-I_{x x}\right)+J_{r} \Omega_{r} \dot{\phi}_{3}+\frac{I_{y y}}{K_{d \theta}} \varepsilon_{\theta} \operatorname{sgn} s_{\theta} \\
& +\frac{I_{y y}}{K_{d \theta}} \eta_{\theta} s_{\theta}
\end{aligned}
$$

\subsubsection{Yaw control}

$$
\begin{aligned}
s_{\psi} & =K_{p \psi}\left(\psi_{d}-\psi\right)+K_{i \psi} \int_{0}^{t}\left(\psi_{d}-\psi\right) d t+K_{d \psi}\left(\dot{\psi}_{d}-\dot{\psi}\right) \\
\dot{s}_{\psi} & =K_{p \psi}\left(\dot{\psi}_{d}-\dot{\psi}\right)+K_{i \psi}\left(\psi_{d}-\psi\right)+K_{d \psi}\left(\ddot{\psi}_{d}-\ddot{\psi}\right) \\
& =-\varepsilon_{\psi} \operatorname{sgn} s_{\psi}-\eta_{\psi} s_{\psi}
\end{aligned}
$$

$$
\begin{aligned}
u_{4}= & \frac{K_{p \psi} I_{z z}}{K_{d \psi}}\left(\dot{\psi}_{d}-\dot{\psi}\right)+\frac{K_{i \psi} I_{z z}}{K_{d \psi}}\left(\psi_{d}-\psi\right) \\
& +I_{z z} \ddot{\psi}_{d}-\dot{\phi} \dot{\theta}\left(I_{x x}-I_{y y}\right)+\frac{I_{z z}}{K_{d \psi}} \varepsilon_{\psi} \operatorname{sgn} s_{\psi} \\
& +\frac{I_{z z}}{K_{d \psi}} \eta_{\psi} s_{\psi}
\end{aligned}
$$

\subsection{Altitude control law}

The motion of the mini-drone quadcopter in the $\mathrm{Z}$-axis is manipulated by the altitude control law as follows [26].

$$
\begin{aligned}
& s_{z}=K_{p z}\left(z_{d}-z\right)+K_{i z} \int_{0}^{t}\left(z_{d}-z\right) d t+K_{d z}\left(\dot{z}_{d}-\dot{z}\right) \\
& \dot{s}_{z}=K_{p z}\left(\dot{z}_{d}-\dot{z}\right)+K_{i z}\left(z_{d}-z\right)+K_{d z}\left(\ddot{z}_{d}-\ddot{z}\right)
\end{aligned}
$$




$$
\begin{aligned}
u_{1}= & \frac{m g}{\cos (\phi) \cos (\theta)}-\frac{m K_{p z}\left(\dot{z}_{d}-\dot{z}\right)}{K_{d z} \cos (\phi) \cos (\theta)} \\
& -\frac{m K_{i z}\left(z_{d}-z\right)}{K_{d z} \cos (\phi) \cos (\theta)}-\frac{m \ddot{z}_{d}}{\cos (\phi) \cos (\theta)} \\
& -\frac{m \varepsilon_{z} \operatorname{sgn} s_{z}}{K_{d z} \cos (\phi) \cos (\theta)}-\frac{m \eta_{z} s_{z}}{K_{d z} \cos (\phi) \cos (\theta)}
\end{aligned}
$$

\subsection{Stability analysis}

The global Lyapunov function for each control law is described as follows [19].

$$
V=V_{0}(s, \dot{s})+\frac{1}{\gamma_{1}}\left(K_{a}-K_{a}^{*}\right) \dot{K}_{a}
$$

where $V_{0}(s, \dot{s})$ is a Lyapunov function. $\gamma_{1}$ is a positive constant, $K_{a}^{*}$ is maximum values of $K_{a}$.

The Lyapunov derivative function (65) is determined as:

$$
\dot{V}\left(s, K_{a}\right) \leq-\eta_{0} \sqrt{V\left(s, K_{a}\right)}+\xi
$$

where $\eta_{0}$ is a positive constant, $V\left(s, K_{a}\right) \geq 0$ is a function of $s, K_{a}$ is adaptation rate.

$$
\xi=-\left|\varepsilon_{K_{a}}\right|\left(\frac{1}{\gamma_{1}} \dot{K}_{a}-\frac{\omega_{1}}{\sqrt{2 \gamma_{1}}}\right)
$$

where $\varepsilon_{s}=K_{a}-K_{a}^{*}, \omega_{1}$ is some a positive constant.

\begin{tabular}{|c|c|c|c|}
\hline Parameters & Symbol & Value & Unit \\
\hline Mass & $m$ & 0.068 & $k g$ \\
\hline Thrust constant & $b$ & 0.0107 & $N s^{2}$ \\
\hline Torque constant & $d$ & $0.783 e-3$ & $N m s^{2}$ \\
\hline Inertial matrix & $\begin{array}{l}I_{x x}, \\
I_{y y}, \\
I_{z z}\end{array}$ & $\begin{array}{c}0.0686 e-3 \\
0.092 e-3 \\
0.1366 e-3\end{array}$ & $\mathrm{~kg} \cdot \mathrm{m}^{2}$ \\
\hline $\begin{array}{c}\text { Distance } \\
\text { between rotor } \\
\text { and center of } \\
\text { mass }\end{array}$ & $l$ & 0.0624 & $m$ \\
\hline
\end{tabular}
This can be shown that the finite time convergence of this method is guaranteed.

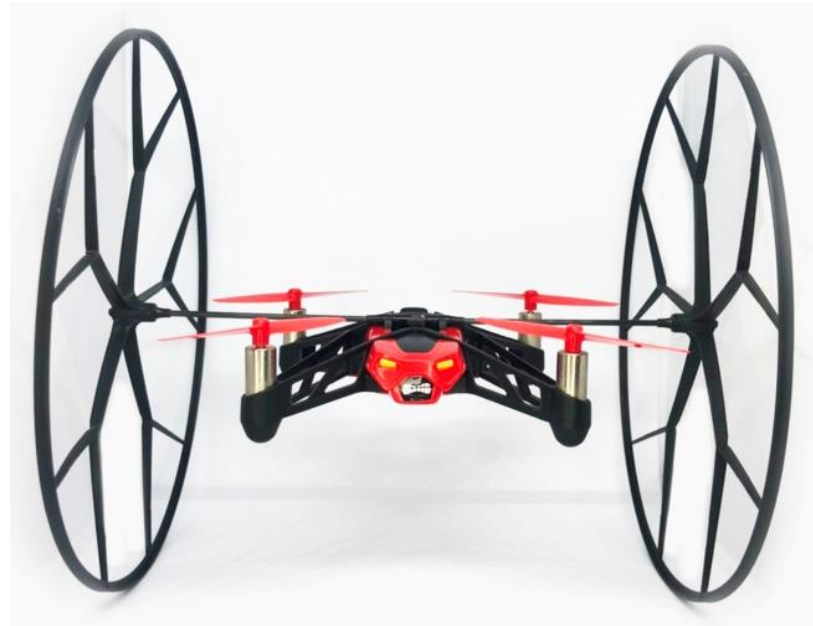

Figure. 4 Parrot rolling spider mini-drone
Table 1. Mini-drone quadcopter parameters

\section{Simulation and results discussion}

In order to verify the efficiency of the proposed MASMC, as explained in section 3, simulations of the parrot roller spider mini-drone quadcopter in Fig. 4 were carried out in 3 cases. The first case covered altitude control, likewise compared with nonadaptive sliding mode control and traditional PID control. The second case covered trajectory tracking control compared with the performance with nonadaptive sliding mode control and traditional PID control. The third case demonstrated the robustness validation for process parameter perturbation and disturbance rejection of MASMC compared with non-adaptive SMC and PID control.

The simulations were developed from a nonlinear model of Mathworks parrot rolling spider mini-drone Simulink support package [27] by increasing the part of trajectory analysis and monitoring, as well as MASMC controller in a flight control system. The physical parameters are depicted in Table 1. The control mechanisms were employed in the flight control system block to validate the performance of the proposed AMSMC compared with SMC and PID control. The objective is to control the mini-drone quadcopter at the specified reference altitude and the $\mathrm{X}-\mathrm{Y}-\mathrm{Z}$ trajectory by minimum overshoot, and the error indexes.

\subsection{Simulation results}

Mini-drone quadcopter in the first case was stabilization at an altitude of 150 centimeters from the initial condition at 0 centimeter.

In the simulation of altitude flight control, the MASMC, SMC, and PID controls were executed in hovering mode at 120 centimeters and 170 centimeters, so that validated the rangeability of the proposed control scheme compared with the others. 


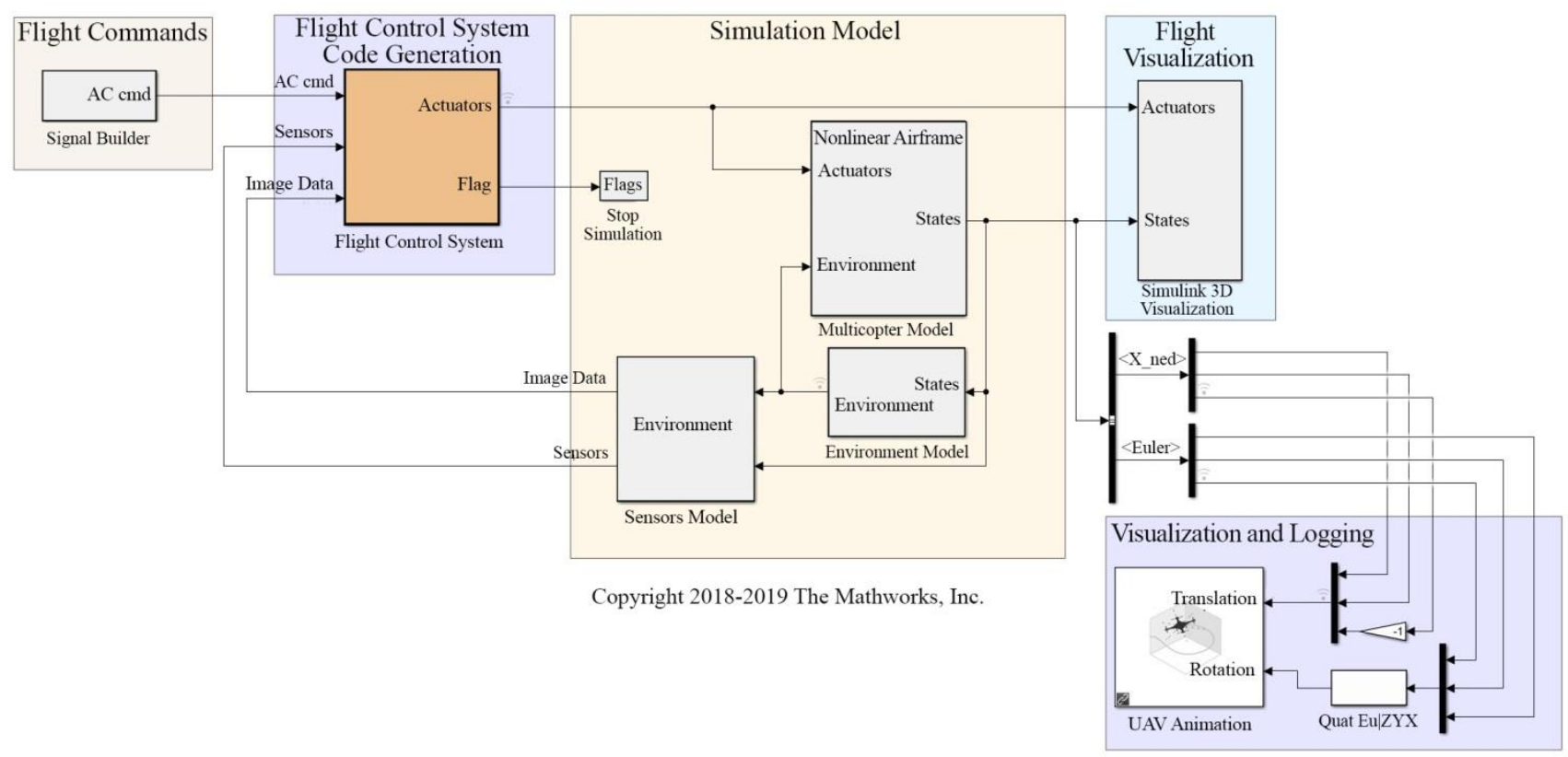

Figure. 5 Quadcopter flight simulation system

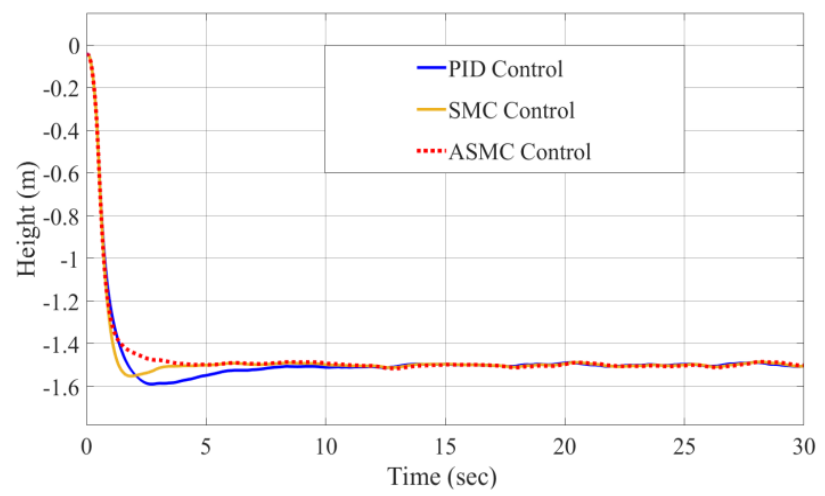

Figure. 6 Step responses of altitude control

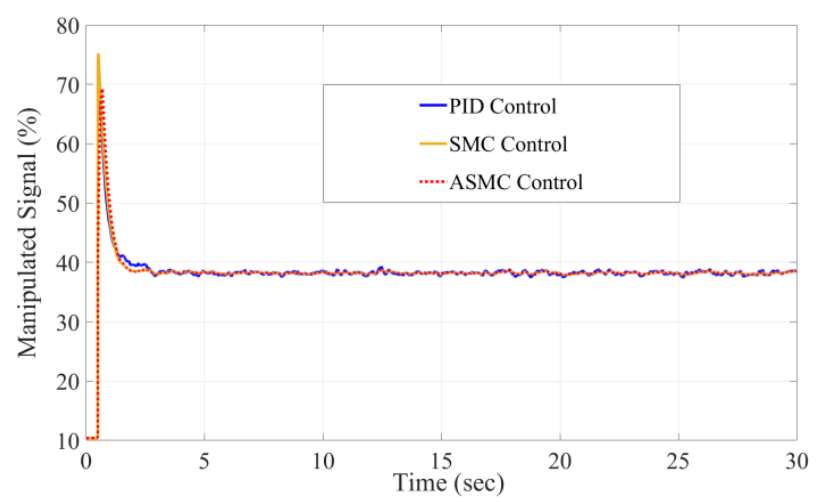

Figure. 7 Control signals of altitude control

For the second case, we performed trajectory tracking. The MASMC, SMC, and PID control were executed in $\mathrm{X}-\mathrm{Y}$ axis trajectory tracking mode at height $(Z) 100$ centimeters moving two rounds along the X-Y axis from $(0,0)$ to $(40,0)(40,40)$ then $(0,40)$ respectively.

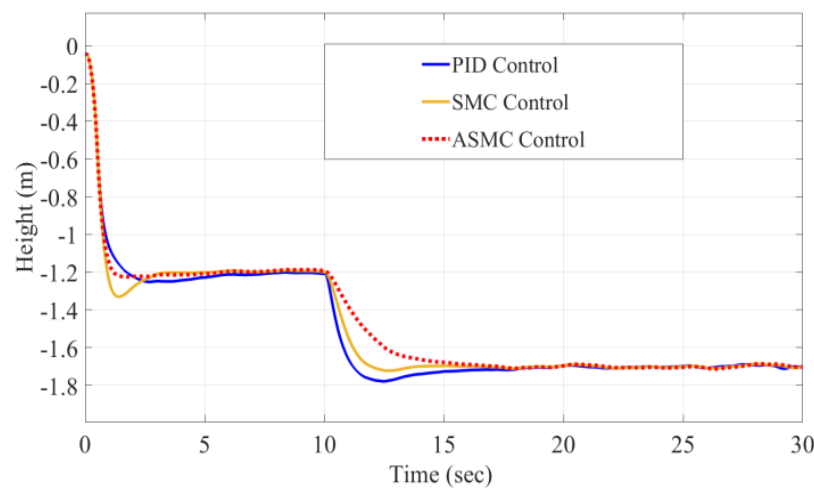

Figure. 8 Step responses of altitude rangeability validation

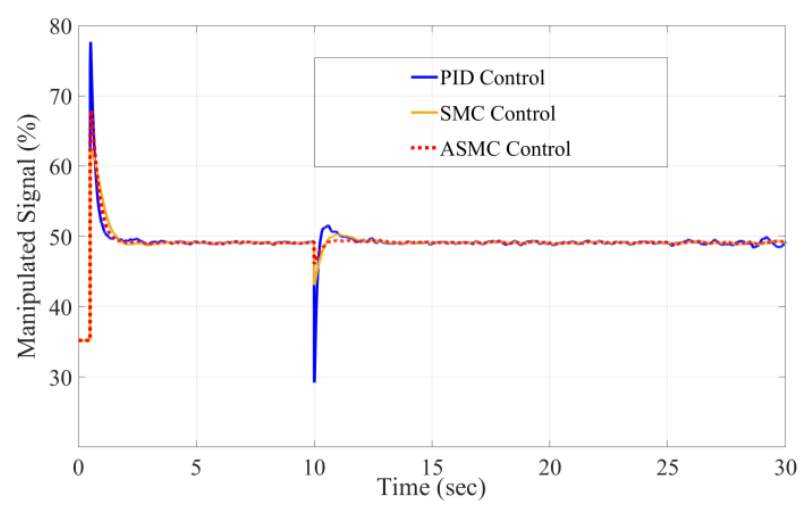

Figure. 9 Control signals of rangeability validation

Compared with the other control scheme, the performance of the proposed MASMC was validated by the performance indexes ISE, IAE, and ITAE. 
Received: April 27, 2019. Revised: June 1, 2020.

Table 2. Performance indexes of altitude control

\begin{tabular}{|c|c|c|c|}
\hline $\begin{array}{c}\text { Performance } \\
\text { indexes }\end{array}$ & MASMC & SMC & PID \\
\hline$\%$ MO & 0, & 3.53, & 6, \\
& $2.08,0$ & $10.83,1.29$ & $4.08,4.64$ \\
\hline Settling time & 3.43, & 3.65, & 7.62, \\
& $2.91,5.35$ & $2.98,3.09$ & $7.16,6.70$ \\
\hline ISE & 1.041, & 1.048, & 1.086, \\
& 0.7331 & 0.8435 & 0.9671 \\
\hline IAE & 1.126, & 1.130, & 1.435, \\
& 1.309 & 1.696 & 1.846 \\
\hline ITAE & 2.611, & 2.942, & 3.623, \\
& 6.872 & 7.945 & 8.423 \\
\hline
\end{tabular}

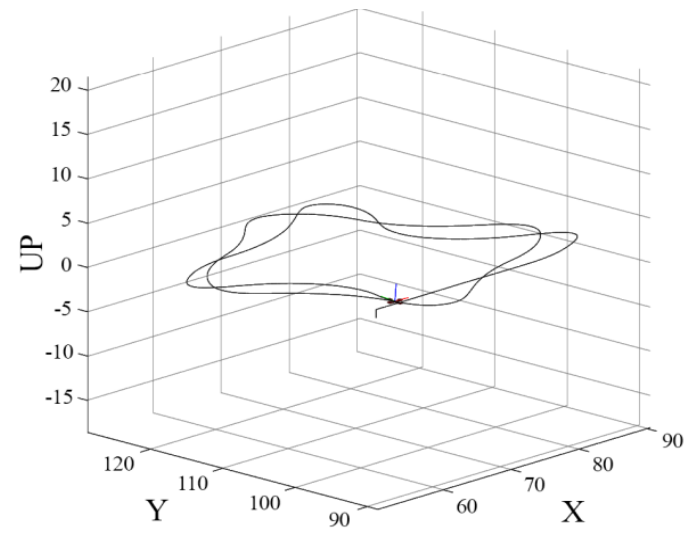

Figure. 10 The response of MASMC trajectory tracking control

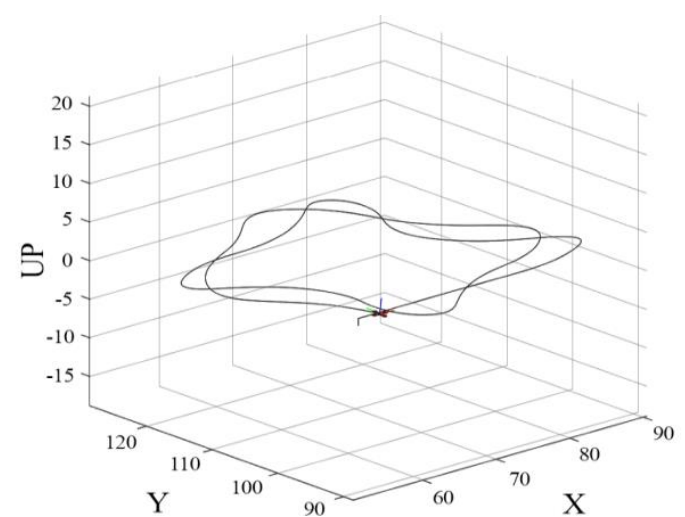

Figure. 11 The response of SMC trajectory tracking control

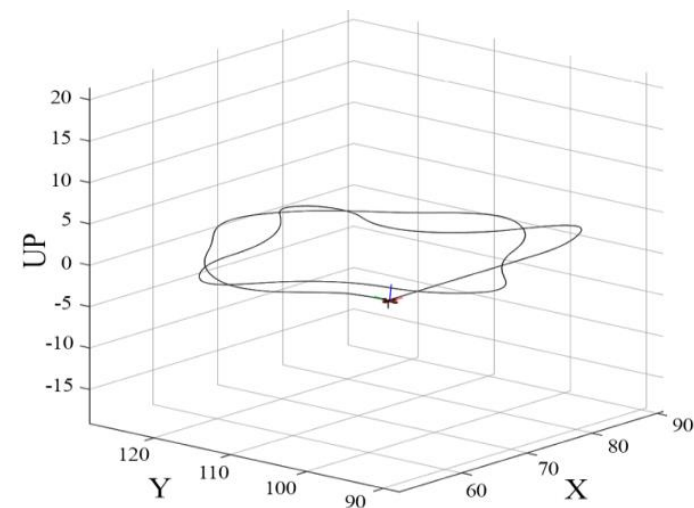

Figure. 12 The response of PID trajectory tracking control

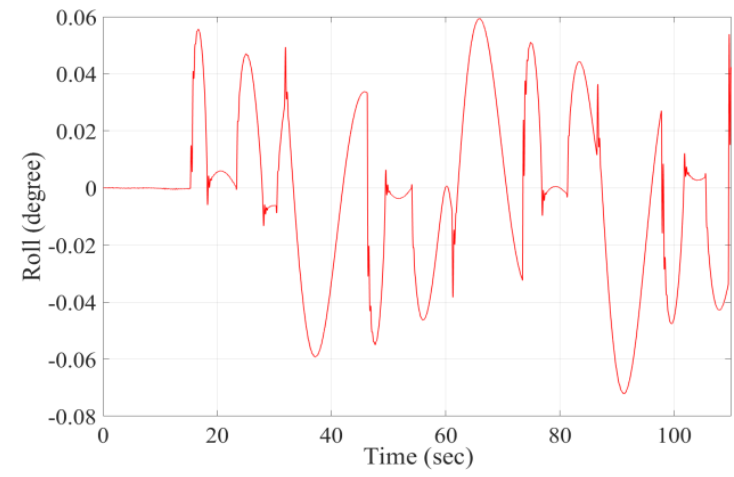

Figure. 13 Roll response of MASMC trajectory tracking control

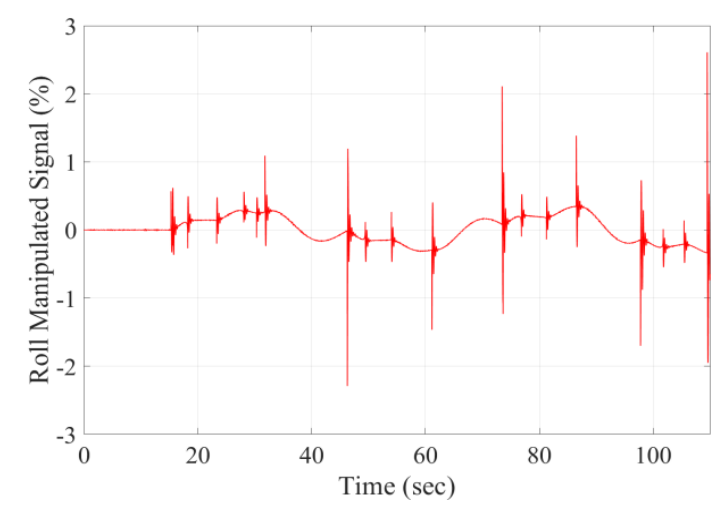

Figure. 14 Roll control signal of MASMC trajectory tracking control

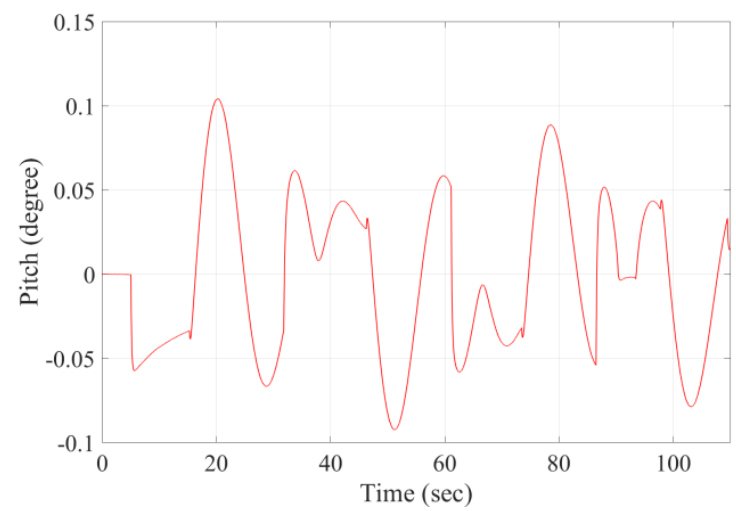

Figure. 15 Pitch response of MASMC trajectory tracking control

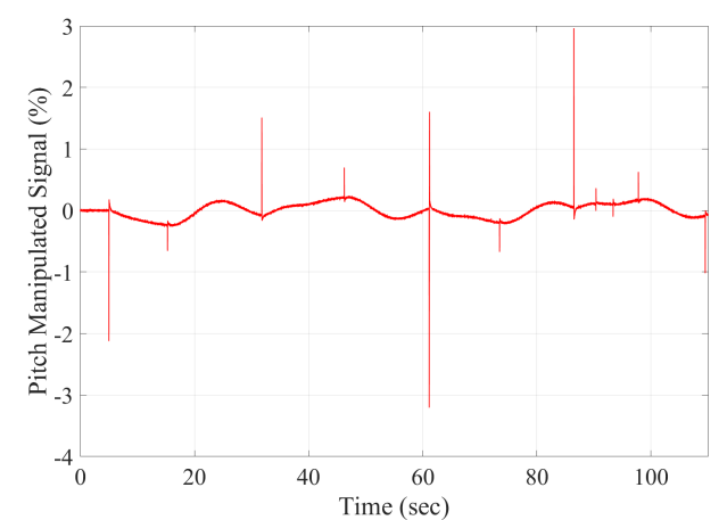

Figure. 16 Pitch control signal of MASMC trajectory tracking control 


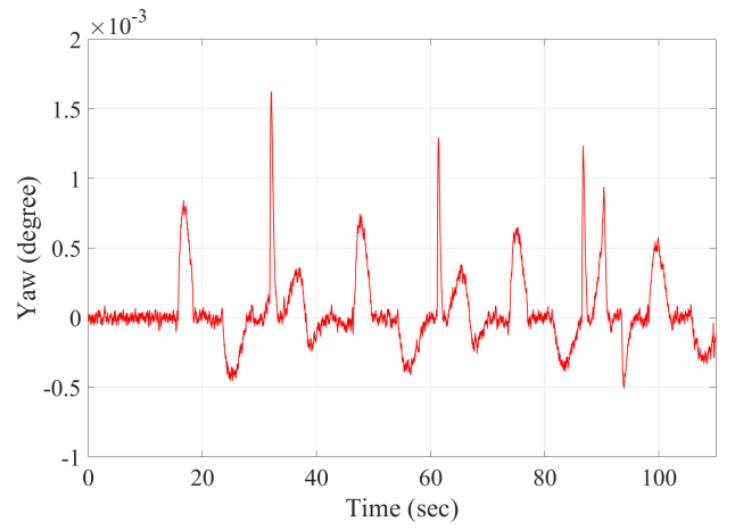

Figure. 17 Yaw response of MASMC trajectory tracking control

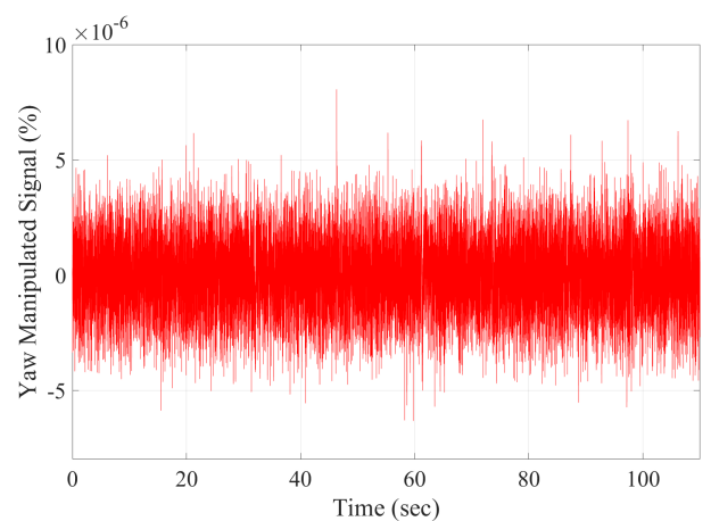

Figure. 18 Yaw control signal of MASMC trajectory tracking control

Table 3. Performance indexes of trajectory control

\begin{tabular}{|c|c|c|c|}
\hline $\begin{array}{c}\text { Performance } \\
\text { indexes }\end{array}$ & MASMC & SMC & PID \\
\hline ISE & 4.091 & 4.626 & 4.72 \\
\hline IAE & 17.14 & 17.97 & 17.85 \\
\hline ITAE & 1032 & 1121 & 1049 \\
\hline
\end{tabular}

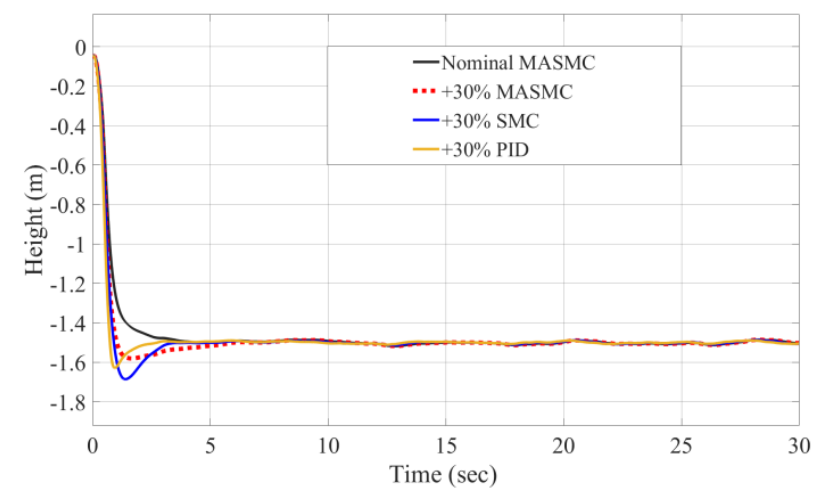

Figure. 19 Step responses at $+30 \%$ process perturbation

The validation of the robustness to process perturbation of the proposed MASMC scheme was performed the altitude and trajectory flight control along with the process gain variation $30 \%$ compared with the other control techniques.

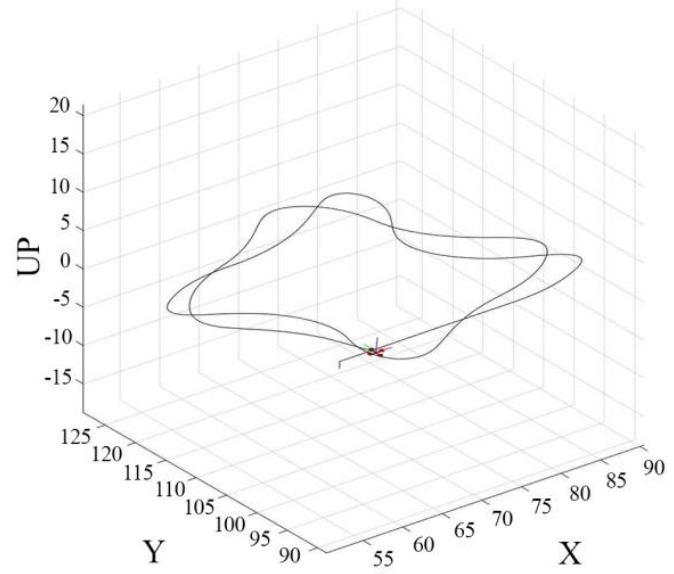

Figure. 20 MASMC trajectory tracking control at $+30 \%$ process perturbation

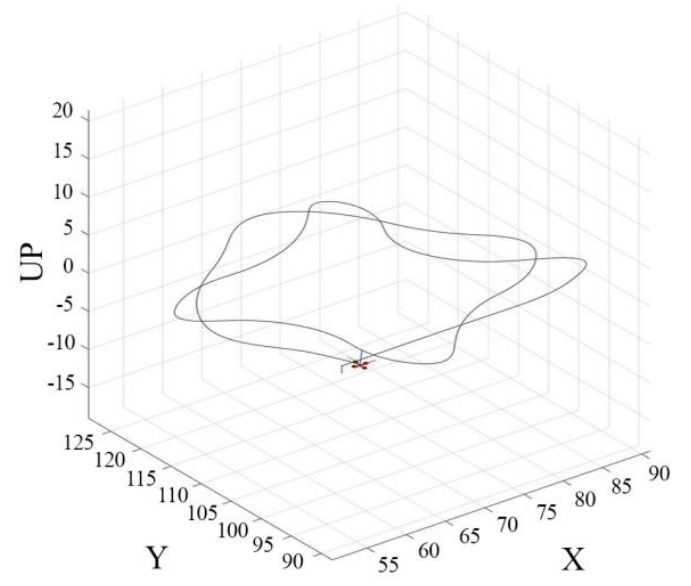

Figure. 21 SMC trajectory tracking control at $+30 \%$ process perturbation

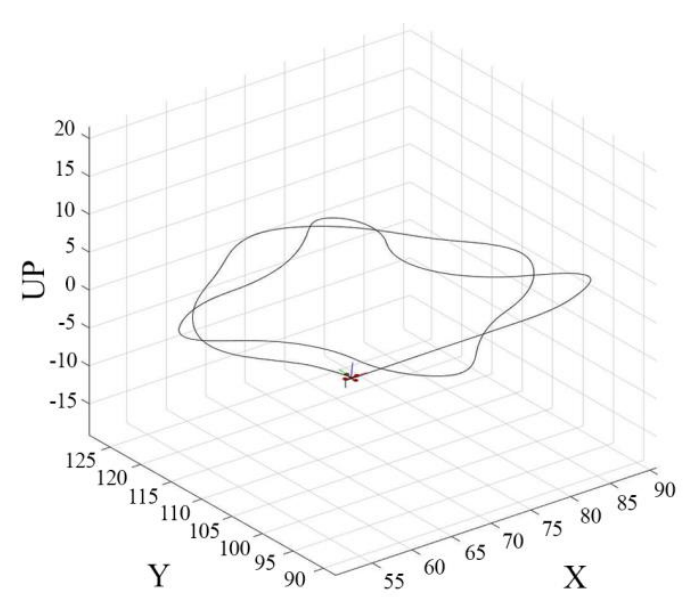

Figure. 22 PID trajectory tracking control at $+30 \%$ process perturbation

The validation of the robustness to the disturbance effect of the proposed MASMC scheme was performed the altitude flight control along with the external force disturbance applied against the height at 1.5 meters of mini-drone compared with the other control techniques. 


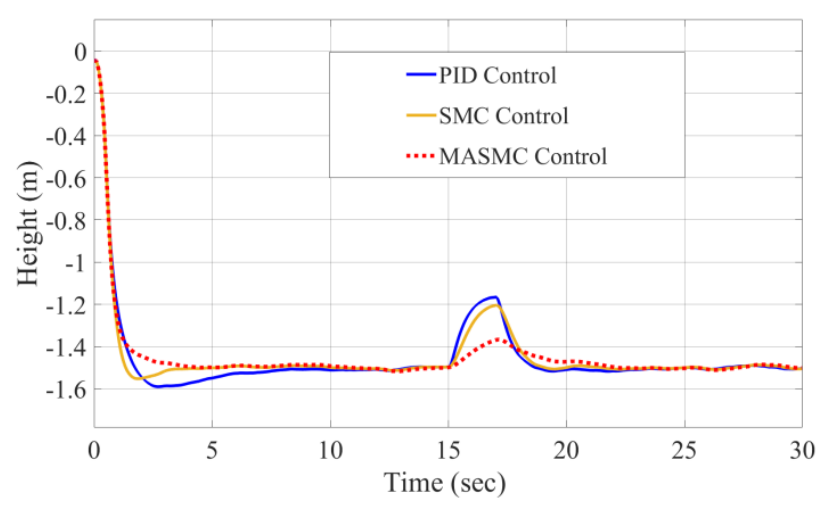

Figure. 23 Step responses for disturbance rejection

Table 4. Performance indexes of robustness validation

\begin{tabular}{|c|c|c|c|}
\hline $\begin{array}{c}\text { Performance } \\
\text { indexes }\end{array}$ & MASMC & SMC & PID \\
\hline $\begin{array}{c}\text { \%OO } \\
\text { perturbation }\end{array}$ & 5.33 & 12.4 & 8.53 \\
\hline $\begin{array}{c}\text { \%O } \\
\text { disturbance }\end{array}$ & 8.8 & 19.53 & 22.2 \\
\hline ISE & 4.231 & 4.985 & 4.723 \\
\hline IAE & 16.64 & 17.67 & 17.81 \\
\hline ITAE & 1051 & 1144 & 1094 \\
\hline
\end{tabular}

\subsection{Discussion}

The results in Fig. 6 exhibited the response of altitude control. It illustrated that MASMC provided the satisfy altitude response less oscillation and settling time, as well as without the steady-state error. In Table 2, the results revealed that MASMC effectively altitudes control of 150 centimeters height with percent overshoot $0 \%$, and the settling time 3.43 seconds. Otherwise, PID control provided less performance by the higher oscillation of the output response in altitude control by the percent overshoot $6 \%$ at the highest settling time of 7.62 seconds.

The results in Fig. 8 illustrated the rangeability of the proposed MASMC technique. The nonlinear characteristic using adaptive gains of MASMC made it has more capable of enabling the excellent control performance of low and high altitude control. The simulation of altitude flight control, the MASMC, SMC, and PID control were executed in hovering mode at 120 centimeters and 170 centimeters. The results demonstrated that MASMC effectively tracking altitudes with percent overshoot $2.08 \%$ and $0 \%$. Otherwise, SMC provided less performance at the percent overshoot $10.83 \%$ and $1.29 \%$. PID provided the percent overshoot at $4.08 \%$ and $4.64 \%$.

The performance indexes, integral of squared error (ISE), integral of absolute error (IAE), and integral of time multiplied absolute error (ITAE) used for indicating the error analysis were shown in Table 2. The results highlighted that MASMC presents the effective performance by providing the lowest error indexes due to the proper nonlinear characteristic of sliding mode control and the adaptive gains; ISE was $1.041 \mathrm{~m}^{2}$, and $0.7331 \mathrm{~m}^{2}$. IAE was $1.126 \mathrm{~m}$, and $1.309 \mathrm{~m}$. ITAE was 2.611 $m \cdot \mathrm{sec}$, and $6.872 \mathrm{~m} \cdot \mathrm{sec}$.

For the second case of trajectory tracking, the MASMC, SMC, and PID control were performed in the $\mathrm{X}-\mathrm{Y}$ axis trajectory tracking mode at height $(\mathrm{Z})$ $100 \mathrm{~cm}$ moving two rounds along the $X-Y$ axis from $(0,0)$ to $(40,0)(40,40)$ then $(0,40)$. The results in Fig. 10-12 presented the response of trajectory tracking control. It clearly showed that the MASMC has a superior performance over the other approaches, such that less deviation path of 2 loop trajectory tracking. Moreover, as shown in Table 3, the results highlighted that MASMC presents the effective performance by providing the lowest error performance indexes due to the proper nonlinear characteristic of sliding mode control and the adaptive gains; ISE is $4.091 \mathrm{~m}^{2}$. IAE is $17.14 \mathrm{~m}$. ITAE is $1032 \mathrm{~m} \cdot \mathrm{sec}$. Fig. 13-18 illustrated the responses and control signals of roll, pitch, yaw, inner attitude control loop, which have been controlled by the MASMC scheme. The results showed that the responses of the rotations in $\mathrm{X}, \mathrm{Y}$, $\mathrm{Z}$-axis are contributed to perform the great response of trajectory tracking.

In addition, the robustness to the effect of process perturbation, the proposed MASMC scheme was validated by performing the altitude and trajectory flight control along with the process gain variation $30 \%$ compared with the other control techniques. The results in Fig. 19-22 showed that the MASMC has superior robustness over the other approaches, such that less percent overshoot $5.33 \%$. At the same time, SMC and PID control provided less performance by the higher oscillation of the output response in altitude control by the percent overshoot $12.4 \%$ and $8.53 \%$, respectively. In table 4 , demonstrated the robustness by the error performance indexes, MASMC presents the effective performance by providing the lowest error indexes due to the proper nonlinear characteristic of sliding mode control and the adaptive gains; ISE was $4.231 \mathrm{~m}^{2}$. IAE was $16.64 \mathrm{~m}$. ITAE was 1051 $m \cdot \mathrm{sec}$.

Furthermore, the robustness to the disturbance's effect, the proposed MASMC scheme was validated by performing the altitude flight control along the external force disturbance applied against the height at 1.5 meters of mini-drone compared with the other 
control techniques. The results in Fig. 23 indicated that the MASMC has an excellent disturbance rejection over the other approaches, such that less oscillation percentage at $8.8 \%$. Otherwise, SMC and PID control provided less robustness by the higher oscillation of the output response by $19.53 \%$ and $22.2 \%$, respectively.

In summary, this study showed that MASMC could be implemented for parrot mini-drone quadcopter effectively by using the adaptive sliding mode control in the outer altitude loop and inner attitude loop, which is the method providing high performance and robustness. The results have proved that the proposed approach has an advantage of performance and robustness control for altitude and trajectory tracking flight control due to nonlinear adaptive mechanism MASMC presents less percent overshoot, as well as provides the smallest error in all the employed performance indexes. On the contrary, SMC and PID control have more oscillation responses, error performance indexes, and less robustness because their nonadaptive and nonlinear control action did not properly perform to control the nonlinear process, including the occurrence of perturbation and disturbance in the quadcopter process. Likewise, using PID-based design, the MASMC algorithm is a less complicated method than other techniques such as the adaptive neural gain scheduling sliding mode control (ANGS-SMC) in [28].

\section{Conclusion}

The modified adaptive sliding mode control (MASMC) for the mini-drone quadcopter unmanned aerial vehicle motion control has been suggested in this study with the purpose of demonstrating the effectiveness of nonlinear adaptive control strategy for achieving the desired performance of mini-drone quadcopter UAV system. The paper describes the mathematical model, and nonlinear dynamic characteristic details of the mini-drone quadcopter actuated system. Furthermore, the modified adaptive sliding mode algorithm has been developed using adaptation law based on the Lyapunov stability approach for suitably applying to altitude and trajectory tracking control. The performance validation of the proposed control technique has been performed on Parrot rolling spider mini-drone using Matlab Simulink program compared with sliding mode control and traditional PID control. The results demonstrated significantly that the nonlinear adaptive mechanism of MASMC could decrease the percentage of overshoot to zero, and reduced the error performance indexes to the minimum ISE at $1.041 \mathrm{~m}^{2}$, IAE $1.126 \mathrm{~m}$, and ITAE $2.611 \mathrm{~m} \cdot \mathrm{sec}$ for altitude. As well as, in the trajectory tracking control, MASMC also provided better ISE performance indexes at $4.091 \mathrm{~m}^{2}$, IAE $17.14 \mathrm{~m}$ of IAE, and $1032 \mathrm{~m} \cdot \mathrm{sec}$ of ITAE. Besides, the MASMC enabled excellent stability and robustness even in the presence of parameter perturbations and disturbance compared to the SMC and PID techniques by providing less maximum overshoot percentage at $5.33 \%$ and $8.8 \%$, reduced the ISE error performance indexes to the minimum of $4.231 \mathrm{~m}^{2}, 16.64 \mathrm{~m}$ of IAE, and $1051 \mathrm{~m} \cdot \mathrm{sec}$ of ITAE. Further, our research intends to integrate the adaptive control mechanism to sensor fusion technology for extending the motion control and application capacity of quadcopter unmanned aerial vehicles.

\section{Acknowledgments}

We gratefully acknowledge the financial support provided by King Mongkut's Institute of Technology Ladkrabang (2562-02-01-021).

\section{Conflicts of Interest}

The authors declare no conflict of interest.

\section{Author Contributions}

J. Chaoraingern and A. Numsomran developed the research methodology, conducted the computations, and verified the proposed methods. V. Tipsuwanporn supported J. Chaoraingern and A. Numsomran to investigate a specific aspect and supervised the results of this work. All authors discussed the results and contributed to the final manuscript.

\section{Nomenclature}

$\begin{array}{ll}R_{e} & \text { rotational matrix } \\ m & \text { mass of quadcopter }(\mathrm{kg}) \\ g & \text { constant of gravitation }\left(\mathrm{m} / \mathrm{s}^{2}\right) \\ T & \text { total thrust force by four propellers }(\mathrm{N}) \\ \omega_{i} & \text { angular velocity }(\mathrm{rad} / \mathrm{s}) \\ I & \text { quadcopter body's inertia tensor }\left(\mathrm{kg} \cdot \mathrm{m}^{2}\right) \\ M_{g h} & \text { gyroscopic moment }(\mathrm{N} \cdot \mathrm{m}) \\ M_{B} & \text { moment from drag forces and thrust }(\mathrm{N} \cdot \mathrm{m}) \\ J_{r} & \text { rotor inertia }\left(\mathrm{kg} \cdot \mathrm{m}^{2}\right) \\ \Omega_{r} & \text { total angular speed of motors }(\mathrm{rad} / \mathrm{s}) \\ d & \text { drag constant }\left(\mathrm{Nms}^{2}\right)\end{array}$


$b \quad$ thrust constant $\left(N s^{2}\right)$

$l \quad$ lever length of the quadcopter's arms $(m)$

$\phi, \theta, \varphi$ roll, pitch and yaw Euler angles ( rad $)$

$\dot{\phi}, \dot{\theta}, \dot{\varphi}$ Euler angular rate $(\mathrm{rad} / \mathrm{s})$

$x, y, z$ distances from quadcopter to the origin of inertial frame $(m)$

$\dot{x}, \dot{y}, \dot{z} \quad$ velocity of quadcopter $(\mathrm{m} / \mathrm{s})$

$\ddot{x}, \ddot{y}, \ddot{z}$ acceleration of quadcopter $\left(\mathrm{m} / \mathrm{s}^{2}\right)$

\section{References}

[1] A. C. Hill, "Economical Drone Mapping for Archaeology: Comparisons of Efficiency and Accuracy", Journal of Archaeological Science: Reports, Vol. 24, pp. 80-91, 2019.

[2] S. S. C. Congress, A. J. Puppala, and C. L. Lundberg, "Total System Error Analysis of UAV-CRP Technology for Monitoring Transportation Infrastructure Assets", Engineering Geology, Vol. 247, pp. 104-116, 2018.

[3] J. Zhang, J. Hu, J. Lian, Z. Fan, X. Ouyang, and W. Ye, "Seeing the Forest from Drones: Testing the Potential of Lightweight Drones as a Tool for Long-term Forest Monitoring", Biological Conservation, Vol. 198, pp. 60-69, 2016.

[4] M. Hassanalian and A. Abdelkefi, "Classifications, Applications, and Design Challenges of Drones: A review", Progress in Aerospace Sciences, Vol. 91, pp. 99-131, 2017.

[5] U. R. Mogili and B. B. V. L. Deepak, "Review on Application of Drone Systems in Precision Agriculture", Procedia Computer Science, Vol. 133, pp. 502-509, 2018.

[6] Y. Karaca, M. Cicek, O. Tatli, A. Sahin, S. Pasli, M. F. Beser, and S. Turedi, "The Potential Use of Unmanned Aircraft Systems (Drones) in Mountain Search and Rescue Operations", The American Journal of Emergency Medicine, Vol. 36, Issue 4, pp. 583588, 2018.

[7] E. Paiva, J. Soto, J. Salinas, and W. Ipanaqué, "Modeling, Simulation and Implementation of a Modified PID Controller for Stabilizing a Quadcopter", In: Proc. of International Conf. On Automatica (ICA-ACCA), Curico, pp. 1-6, 2016.

[8] X. Lu, X. Zhang, S. Jia, and J. Shan, "Design of Quadrotor Hovering Controller Based on Improved Particle Swarm Optimization", In: Proc. of International Symposium on
Computational Intelligence and Design (ISCID), Hangzhou, pp. 414-417, 2017.

[9] C. Liu, J. Pan, and Y. Chang, "PID and LQR Trajectory Tracking Control for an Unmanned Quadrotor Helicopter: Experimental Studies", In: Proc. of International Conf. on Chinese Control Conference (CCC), Chengdu, pp. 10845-10850, 2016.

[10] Z. Shulong, A. Honglei, Z. Daibing, and S. Lincheng, "A New Feedback Linearization LQR Control for Attitude of Quadrotor", In: Proc. of International Conf. On Control Automation Robotics \& Vision (ICARCV), Singapore, pp. 1593-1597, 2014.

[11] E. Kuantama, I. Tarca, and R. Tarca, "Feedback Linearization LQR Control for Quadcopter Position Tracking", In: Proc. of International Conf. On Control, Decision and Information Technologies (CoDIT), Thessaloniki, pp. 204209, 2018.

[12] L. Yanjun, X. Tianqi, and Z. Xiaodong, “A Fuzzy Self-tuning PD Controller for a Quadrotor: Design and Implementation", In: Proc. of International Conf. on Chinese Control and Decision Conference (CCDC), Yinchuan, pp.2448-2453, 2016.

[13] A. Al-Mahturi, F. Santoso, M. A. Garratt, and S. G. Anavatti, "Nonlinear Altitude Control of a Quadcopter Drone Using Interval Type-2 Fuzzy Logic", In: Proc. of International Conf. on IEEE Symposium Series on Computational Intelligence (SSCI), Bangalore, India, pp.236241, 2018.

[14] F. Fakurian, M. B. Menhaj, and A. Mohammadi, "Design of a Fuzzy Controller by Minimum Controlling Inputs for a Quadrotor", In: Proc. of International Conference on Robotics and Mechatronics (ICRoM), Tehran, pp. 619-624, 2014.

[15] B. Lee, H. Lee, and M. Tahk, "Analysis of Adaptive Control Using On-line Neural Networks for a Quadrotor UAV', In: Proc. of International Conference on Control, Automation and Systems (ICCAS), Gwangju, pp.1840-1844, 2013.

[16] Q. Yuan, J. Zhan, and X. Li, "Outdoor Flocking of Quadcopter Drones with Decentralized Model Predictive Control", ISA Transactions, Vol. 71, Part 1, pp. 84-92, 2017.

[17] K. Alexis, G. Nikolakopoulos, and A. Tzes, "Model Predictive Quadrotor Control: Attitude, Altitude and Position Experimental Studies", IET Control Theory \& Applications, Vol. 6, No. 12, pp. 1812-1827, 2012. 
[18] E. H. Zheng, J. J. Xiong, and J. L. Luo, "Second Order Sliding Mode Control for a Quadrotor UAV", ISA Transactions, Vol. 53, No. 4, pp. 1350-1356, 2014.

[19] H. Wang, X. Ye, Y. Tian, G. Zheng, and N. Christov, "Model-free-based Terminal SMC of Quadrotor Attitude and Position", IEEE Transactions on Aerospace and Electronic Systems, Vol. 52, No. 5, pp. 2519-2528, 2016.

[20] S. Nadda and A. Swamp, "Second Order Sliding Mode Control for Quadrotor", In: Proc. of International Conf. on IEEE First International Conference on Control, Measurement and Instrumentation (CMI), Kolkata, pp.92-96, 2016.

[21] N. Ahmed and M. Chen, "Sliding Mode Control for Quadrotor with Disturbance Observer", Advances in Mechanical Engineering, Vol. 10, No. 7, pp. 1-16, 2018.

[22] W. Cai, J. She, and M. Wu, Y. Ohyama, "Disturbance Suppression for Quadrotor UAV Using Sliding-mode-observer-based Equivalent-input-disturbance Approach", ISA Transactions, Vol. 92, pp. 286-297, 2019.

[23] F. Chen, R. Jiang, K. Zhang, B. Jiang, and G. Tao, "Robust Backstepping Sliding-mode Control and Observer-based Fault Estimation for a Quadrotor UAV", IEEE Transactions on Industrial Electronics, Vol. 63, No. 8, pp. 5044-5056, 2016.

[24] X. Gong, Z. C. Hou, C. J. Zhao, Y. Bai, and Y. T. Tian, "Adaptive Backstepping Sliding Mode Trajectory Tracking Control for a Quad-rotor", International Journal of Automation and Computing, Vol. 9, No. 5, pp. 555-560, 2012.

[25] L. R. G. Carrillo, A. E. D. Lopez, R. Lozano, and C. Pegard, Quad Rotorcraft Control: Vision-based Hovering and Navigation, Advances in Industrial Control, Springer, 2013.

[26] J. Liu, and X. Wang, Advanced Sliding Mode Control for Mechanic Systems: Design, Analysis and MATLAB Simulation, Tsinghua University Press, Beijing, Springer, 2012.

[27] Parrot Mini-drones Simulink Support Package, Mathworks, 2019.

[28] N. Cibiraj and M. Varatharajan, "Chattering Reduction in Sliding Mode Control of Quadcopters Using Neural Networks”, Energy Procedia, Vol. 117, pp. 885-892, 2017. 